\title{
Earth tomography with neutrinos in KM3NeT-ORCA
}

\author{
Simon Bourret ${ }^{1}$ and Véronique Van Elewyck ${ }^{1,2, *}$ on behalf of the KM3NeT Collaboration \\ ${ }^{1}$ APC, Université Paris Diderot, CNRS/IN2P3, CEA/Irfu, Observatoire de Paris, Sorbonne Paris Cité, France \\ ${ }^{2}$ Institut Universitaire de France, 75005 Paris, France
}

\begin{abstract}
The study of atmospheric neutrinos crossing the Earth can provide tomographic information on the Earth's interior, complementary to the standard geophysics methods. This contribution presents an updated study of the potential of the KM3NeT-ORCA detector for neutrino oscillation tomography of the Earth, showing that after ten years of operation it can measure the electron density in both the lower mantle and the outer core with a precision of a few percents in the case of normal neutrino mass hierarchy.
\end{abstract}

\section{Neutrino Earth tomography}

Because of their weak interactions, neutrinos offer an alternative way of probing the structure and composition of the inner Earth, complementary to geophysical methods based on the inversion of seismic data, geodetic measurements and laboratory experiments [1]. Neutrinos produced in the interaction of cosmic rays with the atmosphere are particularly appealing for such studies as they span a wide range of energies and pathlengths across the Earth.

At high energies $(>10 \mathrm{TeV})$, because of the rise in cross-section, the Earth starts to become opaque to neutrinos. A study of the angular distribution of neutrinos with increasing energy will reflect the increasing absorption, which is directly related to the amount of matter along the neutrino pathlength [2]. Based on this method, preliminary results on Earth tomography have been obtained recently with a (limited) public sample of IceCube neutrino data [3], showing the potential of the method to constrain the Earth's mass density profile once larger samples will be available.

At lower $(\sim 1-20 \mathrm{GeV})$ energies, atmospheric neutrinos crossing the Earth undergo flavour oscillations which are modified due to coherent forward scattering on electrons $[4,5]$. The signature of these matter effects in the angular, energy and flavour distributions of neutrinos detected at the surface may therefore provide sensitivity to the electron density $n_{e}$ in the different layers of matter traversed. The ratio of electron density $n_{e}$ to mass density $\rho_{m}$ scales with the average proton to nucleon ratio (hereafter denoted $Z / A$ ), which depends on the chemical and isotopic composition of the medium:

$$
n_{e}=\frac{N_{A}}{m_{n}} \times \frac{Z}{A} \times \rho_{m},
$$

where $N_{A}$ is the Avogadro number and $m_{n}$ the nucleon mass. Combining both measurements may thus lead to constraints on the compositional models of the inner Earth [6]. In this contribution, we present the latest estimates on the sensitivity of the KM3NeT-ORCA detector to the $Z / A$ ratio in the mantle and outer core, assuming the reference geophysical model (PREM [7]) for the radial mass density profile.

\footnotetext{
*e-mail: elewyck@apc.univ-paris7.fr
} 
Table 1. Compositional layers in the Earth model used in the analysis. The columns indicate the number of constant density shells, the exact innermost and outermost radius, and the assumed $\mathrm{Z} / \mathrm{A}$ value.

\begin{tabular}{llll} 
Layer & Shells & {$\left[R_{-}, R_{+}\right](\mathrm{km})$} & $Z / A$ \\
\hline Inner core & 4 & $0-1221.5$ & 0.4691 \\
Outer core & 20 & $1221.5-3480$ & 0.4691 \\
Lower mantle & 11 & $3480-5701$ & 0.4954 \\
Upper mantle & 5 & $5701-6346$ & 0.4954 \\
Crust & 2 & $6346-6368$ & 0.4956 \\
Water & 1 & $6368-6371$ & 0.5525 \\
Atmosphere & 1 & $6371-6386$ & 0.4991 \\
\hline
\end{tabular}

\section{The KM3NeT-ORCA detector and simulation}

ORCA (Oscillations Research with Cosmic in the Abyss) is the low-energy branch of KM3NeT [8], the next-generation water-Cherenkov neutrino detector currently under construction in the Mediterranean Sea. ORCA is being deployed off the French coast, at $2450 \mathrm{~m}$ depth, and is dedicated primarily to fundamental neutrino physics studies, with the main objective of measuring the neutrino mass hierarchy [9]. The detector will comprise 115 vertical strings, with an horizontal spacing of $\sim 23 \mathrm{~m}$. The key detection element is the Digital Optical Module (DOM), a glass sphere comprising 31 photomultipliers oriented towards all directions. Each string supports 18 DOMs with a vertical spacing of $\sim 9 \mathrm{~m}$, instrumenting a total mass of about 8 Mton of seawater. With this configuration, ORCA will focus on the study of atmospheric neutrino oscillations in the energy range $\sim 1-100 \mathrm{GeV}$.

The analysis presented here uses a full Monte Carlo simulation of the detector, that includes neutrino-nuclei interactions, propagation and decays of secondary particles, Cherenkov light generation and propagation, photo-detection and electronics effects. The event topologies of neutrino interactions with nuclei in seawater in the energy range of interest for ORCA can be separated in two broad classes: track-like events are associated to $v_{\mu}$ (or $v_{\tau}$ ) charged-current interactions producing a muon that crosses the detector, while cascadelike events correspond to all other (charged- and neutral-current) interaction channels, where only hadronic and electromagnetic showers are produced. Dedicated event reconstruction algorithms have been developed for both track-like and cascade-like signatures. Event classification, together with rejection of down-going atmospheric muons, are based on a machine learning technique. Details regarding simulations, detector resolutions and classification performance can be found in [8].

\section{Sensitivity of ORCA for neutrino oscillation tomography}

The analysis relies on the computation of energy $E$ and zenith angle $\theta_{z}$ distributions of tracklike and cascade-like events originating from interactions of atmospheric neutrinos crossing the Earth (see top left panel of Fig. 1). The tools and inputs used to simulate the neutrino propagation, oscillation and interaction are described in [10]. The Earth electron density is a radial model with 44 concentric shells of constant electron density, where mass density values are fixed and follow the PREM. Additionally, seven chemical layers are defined, where the composition, and hence the $Z / A$ factor, are assumed to be uniform (see Table 1).

The detector response is modeled using a full Monte Carlo approach. For each discrete channel (specified by the neutrino polarity $v / \bar{v}$, flavour, and event classification) a dedicated binned 4-dimensional response matrix is used for the transformation $\left(E_{\text {true }}, \theta_{\text {true }}\right) \rightarrow$ $\left(E_{\text {reco }}, \theta_{\text {reco }}\right)$. These matrices are built using Monte Carlo generated events and the outcome of their processing through the reconstruction and classification algorithms, so that the ensemble of matrices accounts for detection and reconstruction efficiencies, misidentification probabilities and errors on reconstructed variables (including all correlations). 


\begin{tabular}{lllll} 
parameter & treatment & true value & prior & $1 \sigma$ width \\
\hline $\operatorname{sign}\left(\Delta m_{31}\right)$ & fix & NH or IH & - & - \\
$\left|\Delta m_{31}^{2}\right|_{\mathrm{NH}}\left(\mathrm{eV}^{2}\right)$ & fitted & $2.49410^{-3}$ & no & - \\
$\left|\Delta m_{31}^{2}\right|_{\mathrm{IH}}\left(\mathrm{eV}^{2}\right)$ & fitted & $2.39110^{-3}$ & no & - \\
$\Delta m_{21}^{2}\left(\mathrm{eV}^{2}\right)$ & fix & $7.4010^{-5}$ & - & - \\
$\theta_{13}\left({ }^{\circ}\right)$ & fitted & 8.54 & yes & 0.15 \\
$\theta_{12}\left({ }^{\circ}\right)$ & fix & 33.62 & - & - \\
$\theta_{23}\left({ }^{\circ}\right)$ & fitted & 45 & no & - \\
$\delta_{\mathrm{CP}}\left({ }^{\circ}\right)$ & fitted & 0 & no & - \\
\hline
\end{tabular}

Table 2. Treatment of oscillation parameters in this analysis.

The potential of ORCA for probing Earth's electron density has been studied by considering the measurement of uniform variations (rescaling) of the electron density in whole compositional layers. The measurements of greater interest for geophysics are those of the lower mantle and outer core, therefore we have focused our study on these two layers and have included the other layers as fixed parameters. Since ORCA will be capable of determining the neutrino mass ordering with a confidence level of more than $3 \sigma$ in 3-4 years [9], and considering that the Earth tomography measurements need a significantly longer data-taking duration, the mass ordering can be assumed as already known and is kept fixed in the analysis.

To compute the final sensitivity of ORCA, we use a binned log-likelihood ratio method based on Asimov datasets, where the $\overline{\Delta \chi^{2}}$ test statistic is profiled over the ensemble of nuisance parameters and external constraints are accounted for under the form of gaussian "prior" likelihood terms for some parameters (see [10] for more detail). In the fitting process of a given layer, the incomplete knowledge of the other layer $\mathrm{Z} / \mathrm{A}$ is also considered as a source of systematic uncertainty. This study provides an improved treatment of statistics with respect to [10]; it includes in particular the correction of a statistical artifact induced by limited Monte Carlo statistics (or "sparse Monte Carlo effect"), which has been shown to lead to an overestimation of the sensitivity [11].

The right panels of Fig. 1 shows the $\overline{\Delta \chi^{2}}$ profiles for the outer core and lower mantle measurements within the region of interest for the $Z / A$ parameter, with and without including the systematic uncertainties and assuming either normal or inverted neutrino mass ordering. For all curves the $Z / A$ scaling in the other layer is profiled upon as a systematic, and the sparse Monte Carlo effect is corrected for. Additional nuisance parameters are added incrementally, according to the settings of Table 2: (i) addition of $\theta_{23}, \Delta m_{31}^{2}$ and $\delta_{\mathrm{CP}}$ (free); (ii) addition of $\theta_{13}$ (standard prior) and the full set of systematics: $\mu / e$ flavour skew (5\% prior), $v_{e} / \bar{v}_{e}$ skew (5\% prior), $v_{\mu} / \bar{v}_{\mu}$ skew (5\% prior), neutral-current events normalisation (10\% prior), global energy and zenith slope parameters on the neutrino flux (free). In addition, two-dimensional contours for the combined measurement of both $Z / A$ parameters are shown in the bottom left panel of Fig. 1.

All systematics included and assuming true $\mathrm{NH}$, a level of precision of approximately $\pm 5 \%$ can be reached in the lower mantle, and $\pm 7 \%$ in the outer core, both at $1 \sigma$ confidence level (C.L.). The inverted hierarchy is much less favorable, mostly due to the fact that the MSW resonance then occurs for antineutrinos, whose charged-current cross-section is about twice smaller than for neutrinos. The measurement in the outer core is not strongly correlated with that in the mantle, demonstrating the ability of the technique to access the inner Earth without being affected by the shielding of the outermost layers. The hypothesis of identical chemical compositions of the lower mantle and outer core could be excluded at a $1 \sigma$ C.L., as could exotic compositional models, such as the hydridic Earth model predicting an average $Z / A \sim 0.57$ in the core [12]. These results are in agreement with previous theoretical studies [13] and illustrate the concrete potential of neutrino oscillation tomography as a com- 
pletely new technique, based solely on the weak interaction of neutrinos. Increasing ORCA's (or any future detector's) performances for this measurement would likely require to lower its detection threshold and to increase its reconstruction and classification performance at low $(\sim 1 \mathrm{GeV})$ energy.
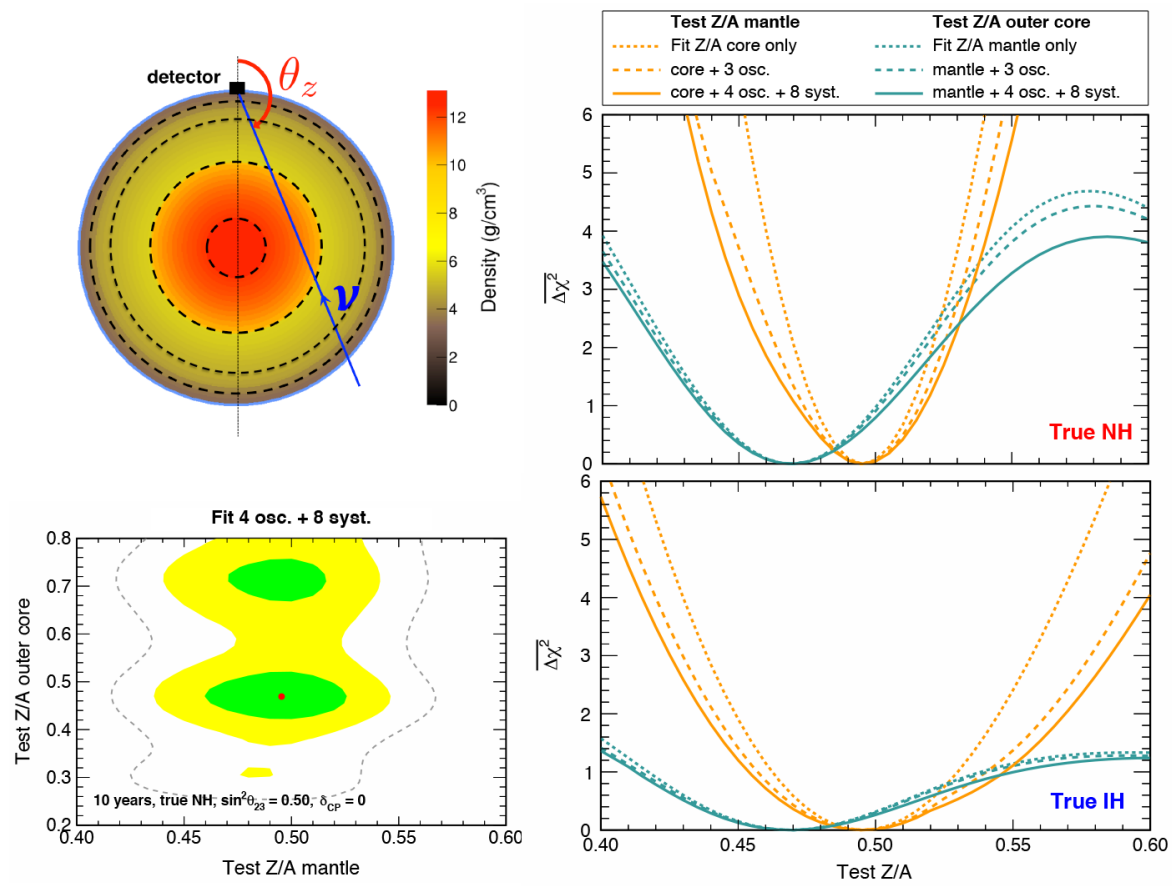

Figure 1. Top left: Density profile of the PREM (color scale) and compositional layers as in Table 1 (dashed). Bottom left: Expected confidence regions for the combined measurement of the Z/A scaling in the lower mantle and outer core (green: $1 \sigma$, yellow: $2 \sigma$, dashed: $3 \sigma$ ). Right: One-dimensional profile likelihood ratio for the measurement of the Z/A scaling in the lower mantle and outer core after 10 years of operation of ORCA. The top (bottom) panel correspond to a true normal (inverted) hierarchy. In both cases $\sin ^{2} \theta_{23}=0.5$, and $\delta_{\mathrm{CP}}=0$.

\section{Acknowledgements}

The authors gratefully acknowledge financial support from LabEx UnivEarthS at Sorbonne Paris Cité (ANR-11IDEX-0005-02, ANR-10-LABX-0023 and from the Agence Nationale de la Recherche (ANR-15-CE31-0020).

\section{References}

[1] see e.g. the review by W. Winter, Earth Moon Planets 99285 (2006).

[2] M. C. Gonzalez-Garcia et al., Phys. Rev. Lett. 100061802 (2008); E. Borriello et al., JCAP 0906030 (2009).

[3] A. Donini, S. Palomares-Ruiz and J. Salvado, Nature Phys. 15 no.1, 37 (2019).

[4] S. Mikheev and A. Smirnov, Sov. J. Nucl. Phys. 42913 (1985); L. Wolfenstein, Phys. Rev. D 172369 (1978).

[5] S. Petcov, Phys. Lett. B434 321 (1998); M. Chizhov and S. Petcov, Phys. Rev. D63 073003 (2001).

[6] C. Rott, A. Taketa and D. Bose, Sci.Rep. 515225 (2015); W. Winter, Nucl.Phys. B908 250 (2016).

[7] M. Dziewonski and D. L. Anderson, Physics of the Earth and Planetary Interiors 25297 (1981).

[8] S. Adrian-Martínez et al. [KM3NeT Coll.], J. Phys. G: Nucl. Part. Phys. 43084001 (2016).

[9] P. Coyle [KM3NeT Coll.], Status of ORCA and ARCA, this Conference.

[10] S. Bourret et al. [KM3NeT Coll.], PoS ICRC2017 1020 (2018).

[11] S. Bourret, Neutrino oscillations and Earth tomography with KM3NeT-ORCA, Ph. D. Thesis, Université Paris Diderot - Sorbonne Paris Cité (2018), in press.

[12] L Bezrukov and V Sinev. "Atmospheric neutrinos for investigation of Earth interior", In: Physics of Particles and Nuclei 47.6, 915 (2016).

[13] W. Winter, Nucl. Phys. B908 250 (2016). 